Bettina Blanck

Erwägungsorientierung, Entscheidung und Didaktik 


\section{Erwägungskultur in Forschung,}

\section{Lehre und Praxis}

(herausgegeben von Werner Loh)

\section{Band 2}

Menschheitsgeschichtlich bedacht sind in den letzten Jahrhunderten - vom Mikrobereich der Atome bis zum Makrobereich des Weltraums, von der Gentechnik bis zur Robotertechnik - reproduzierbare und radikal neue Ergebnisse gewonnen worden. Trotzdem bestehen in den Wissenschaften nicht nur hinsichtlich ihrer Grundlagen einander widersprechende Auffassungen - von der Mathematik über Physik und Biologie bis hin zu den Kulturwissenschaften -, sondern auch darüber, wie mit diesen Ergebnissen praktisch umgegangen werden sollte. Viele dieser Differenzen sind in Weltbildern verankert, die zuweilen über mehrere tausend Jahre zurück verfolgbar sind. Es gibt bisher keine Tradition, die derartige Differenzen erforschend in Erwägungen einzubringen trachtet. Erwägen ist ein konstitutiver Bestandteil menschlicher Problembewältigung als Entscheidungsprozess. Erwägungen können erinnert und damit auch als Geltungsbedingungen von Lösungen bewahrt und verbessert werden, insbesondere für Erwägungen, wie zu erwägen sei; hierdurch werden Erwägungsforschungsstände möglich. In der Reihe Erwägungskultur in Forschung, Lehre und Praxis werden Arbeiten veröffentlicht, die sich am Konzept des Erwägens orientieren. Es werden sowohl Grundlagen als auch spezifische Anwendungsfragen behandelt. Methodisch reicht das Spektrum von der Zusammenführung unterschiedlicher Auffassungen, die zu Erwägungen herausfordern, bis hin zu kombinatorischen Vorgehensweisen. Die Reihe soll Tradierungen ermöglichen, die der Relevanz entsprechend sich in Forschung, Lehre und Praxis methodisch um Verbesserungen von Erwägungen umfassend kümmern. 


\section{Erwägungsorientierung, Entscheidung und Didaktik}

von Bettina Blanck

mit 41 Übersichten und Diagrammen

$\underset{\infty}{\longrightarrow} \quad$ Lucius und Lucius 
Anschrift der Autorin:

Bettina Blanck

Univ.-GH Paderborn

FB 1

Warburger Str. 100

33098 Paderborn

Die Deutsche Bibliothek - CIP-Einheitsaufnahme

Erwägungsorientierung, Entscheidung und Didaktik / von Bettina Blanck. - Stuttgart : Lucius und Lucius, 2002

(Erwägungskultur in Forschung, Lehre und Praxis ; Bd. 2) ISBN 3-8282-0208-X

Zugl.: Paderborn, Univ., Diss. 2001

(C) Lucius \& Lucius Verlagsgesellschaft mbH, Stuttgart 2002

Gerokstr. 51, D-70184 Stuttgart

Das Werk einschließlich aller seiner Teile ist urheberrechtlich geschützt. Jede Verwertung außerhalb der engen Grenzen des Urheberrechtsgesetzes ist ohne Zustimmung des Verlages unzulässig und strafbar. Das gilt insbesondere für Vervielfältigung, Übersetzungen, Mikroverfilmungen und die Einspeicherung, Verarbeitung und Übermittlung in elektronischen Systemen.

Druck und Einband: Rosch-Buch, Scheßlitz

Printed in Germany 